Gazi University
Journal of Science
http://dergipark.gov.tr/gujs

\title{
SPICE Model of Current Polarity-Dependent Piecewise Linear Window Function for Memristors
}

\author{
Ertugrul KARAKULAK ${ }^{1, *(1)}$, Resat MUTLU2 ${ }^{(1)}$ \\ ${ }^{I}$ Tekirdă̆ Namık Kemal University, Vocational School of Technical Sciences, Electronics Department, Tekirdağ, Turkey \\ ${ }^{2}$ Tekirdă̆ Namık Kemal University, Çorlu Engineering Faculty, Electronics and Communication Engineering Dep. Çorlu, Tekirdağ, Turkey
}

\section{Highlights}

- A novel window function for Memristors based on PWL Functions.

- Current polarity dependent window function for Memristor.

- A Memristor model for LTSPICE simulator.

- Simulation results of window function for Memristors based on PWL Functions.

\section{Article Info}

Received: $12 / 08 / 2019$

Accepted: 26/04/2020

Keywords

Memristor

Piecewise linear functions

Window functions

Spice model

\begin{abstract}
Memristor and memristive systems are nonlinear systems. It is important to model them accurately. There are different memristor models and most of the models make use of window functions. In literature, there are various window functions. Recently, a piecewise linear (PWL) window function is used to model a memristor and memristive systems. Such a memristor with a PWL window function lacks a SPICE model. Also, in literature, there is current polarity dependent window functions proposed for memristors to model polarity dependent drift speed within the thin-film memristors. In this study, an alternative current-polarity dependent PWL window function is suggested to model a memristor, a different PWL function one for each current polarity is used, its SPICE model is made in LTSpice and also its simulation results are given. Such a model can be used to model the polarity dependent drift speed within the thin-film memristors.
\end{abstract}

\section{INTRODUCTION}

In 1971, Memristor has been theoretically claimed to exist by Dr. Leon Chua [1]. In 1976, memristive systems with similar properties to memristors have been described [2]. In 2008, a research team has announced that thin-film memristive system which behaves as if a memristor has been found [3]. In the last decade, memristor and memristive systems have become a hot research area [4-9]. That's why it is important to develop memristor models. Nowadays, window functions are commonly used to model memristors and different window functions are available in the literature [3, 10-14]. The first window function is given by Strukov et al [3]. Joglekar has also given a nonlinear dopant drift memristor model [10]. Prodromakis et al have given a continuous and scalable window function [11]. Biolek et al have given a current direction dependent window function [13]. Zha et al have modified Prodromakis model to get rid of the boundary tackling issue and to allow scalability [14]. The models in [10-12] have boundary lock problem. All the models are phenomenological approaches. A model has to be flexible enough to mimic the experimental data given in the literature. In literature, piece-wise linear (PWL) flux-charge memristor characteristics have been previously used first by Chua in 1971 [1] and then by also others in memristorbased chaotic oscillators and filter studies [15-20]. A memristor model which makes use of a PWL window function model is suggested in [21]. It shows an alternative to model memristors without using the continuous window functions given in [10-14].

Because of non-linear nature, it is hard to analyze the electrical characteristic of the memristors. Memristance of memristors change with respect to the state variable x. Derivative of the state variable $\mathrm{x}$ 
depends on the window function and memristor current. Memristors are nonlinear circuit elements and their window functions are also of nonlinear nature. If a PWL state variable function is used, such a nonlinear function can be segmented limited number of pieces and every piece can be evaluated as linear functions. If the number of linear pieces are high enough, a PWL window function approximates the nonlinear function [21-22]. Biolek and Zha window functions have current polarity dependent [13-14]. Due to their current dependency, these models eliminate the boundary tackling issues some memristor models suffer from $[3,10-12]$.

In this paper, a novel current direction dependent PWL window function is proposed model memristors. Such a model may help to increase the modelling accuracy of the memristors. The model can be made more accurate by increasing the number of PWL sections. The new PWL function is flexible and it is able to model both symmetric and asymmetric Lissajous curves of memristors. In addition, LTSpice code of proposed model is given.

This paper is arranged in the following way. In the second section, memristive systems are briefly introduced. In the third section, the new current-dependent PWL window function is given. In the fourth section, its Spice model is given. In the fifth section, the simulation results are given. The results are discussed in the conclusion section.

\section{MEMRISTOR MODELS AND DIFFERENT WINDOW FUNCTIONS}

\subsection{Memristive Systems}

In [6], Ventra and et al. has described nth degree current-controlled memristive sytems as,

$v(t)=R[x(t), i(t), t] \cdot i(t)$,

$\frac{d x}{d t}=f[x(t), i(t), t]$

where $v(t)$ is the voltage of the memristive system, $i(t)$ is the current of the memristive system. $x(t)$ is an $\mathrm{n}$ dimensional state variable vector, $R$ is the resistance of the memristive system which is dependent on $i(t)$ and $x(t)$.

\subsection{Thin-film Memristive Systems}

An (ideal) memristor is a special case of the memristive systems [1]. However, thin-film memristive systems which are actually memristive systems are also called memristors nowadays. Such a memristor model with nonlinear dopant drift is given in Equations (3) and (4),

$v(t)=R(x) i(t)$,

$\frac{d x}{d t}=\mu_{v} \frac{R_{O N}}{D^{2}} \cdot i(t) f(x)$

where $R(x)$ is the memristor resistance, $i(t)$ is its current, $v(t)$ is its voltage, $w$ is its oxidized length, $D$ is the total length of $\mathrm{TiO}_{2}$ region, $x=w / D$ is its normalized oxidized length, $\mu_{v}$ is its dopant mobility, $R_{O N}$ is its minimum resistance and $f(x)$ is its window function.

The $\mathrm{TiO}_{2}$ memristor can be written as,

$R(x)=R_{o f f}-\left(R_{o f f}-R_{o n}\right) x$. 
Its resistance ranges from its minimum value $\mathrm{R}_{\mathrm{ON}}$ to its maximum value $\mathrm{R}_{\mathrm{OFF}}$. A window function is an indication of how much a memristive system approaches to being an ideal memristor [3]. Their resistance value or memristive state-variable starts changing only when the window function $f(x)$ is different from zero. Some well-known memristor window functions are given in Table 1 . The window functions in [3,1012] have zero dopant speeds at the memristive layer boundaries called boundary tackling issues: at $x=0$ and $x=1$. Their resistance value or memristive state-variable or window function $f(x)$ does not change whatever the current is. The model in [13-14] do not suffer from the issues. Those window functions given in Table 1 are all phenomenal functions. Experimental data may need a more realistic window function for a better accuracy.

Table 1. Some memristor window functions

\begin{tabular}{|l|l|}
\hline Window Function Name & Window Functions \\
\hline Strukov [3] & $f(x)=x-x^{2}$ \\
\hline Joglekar [10] & $f(x)=1-(2 x-1)^{2 p}$ \\
\hline Prodromakis [11] & $f(x)=j\left(1-\left((x-0.5)^{2}+0.75\right)^{p}\right)$ \\
\hline Oğuz [12] & $f(x)=j\left(e^{-\left(\frac{x-0.5}{c}\right)^{2 p}}-e^{-\left(\frac{0.5}{c}\right)^{2 p}}\right)$ \\
\hline Biolek [13] & $f(x)=1-(x-\operatorname{stp}(-i(t)))^{2 p}$ \\
\hline Zha [14] & $f(x)=j\left(1-\left(0.25(x-\operatorname{stp}(-i))^{2}+0.75\right)^{p}\right)$ \\
\hline
\end{tabular}

\section{MEMRISTOR MODEL WITH THE PWL WINDOW FUNCTIONS}

\subsection{Chua's PWL Function Model}

In [21], a PWL window function is used to model a memristor. The model uses Chua's PWL function as memristor window function. Generic formulation of the PWL function is proposed by Leon Chua et al. using well-known canonical equation [22] is,

$$
f(x)=\propto+B x+\sum_{i=1}^{\sigma} C_{i}\left|x-x_{i}\right| .
$$

Parameters $\alpha, B$ and $C_{i}$ can be calculated using Equations (7)-(9)

$$
\begin{aligned}
& B=\frac{\left(J^{(1)}+J^{(\sigma)+1}\right)}{2}, \\
& C_{i}=\frac{\left(J^{(i+1)}-J^{i}\right)}{2}, i=1,2, \ldots \sigma, \\
& \alpha=f(0)-\sum_{i=1}^{\sigma} C_{i}\left|x-x_{i}\right| .
\end{aligned}
$$

\subsection{Current-polarity Dependent Memristor Model with The PWL Window Functions}

In [16-21], PWL window functions are used to model memristors. An alternative writing format of a PWL function is also given in this section. Let's assume that a window function designated as $f(x)$ can be approximated piecewise linearly as $g(x)$. The PWL function $g(x)$ consists of $\mathrm{n}$-segments and $\mathrm{n}$-lines (slopes) 
as shown in Figures 1 and 2. The state variable, the window function $f(x)$ and PWL function $g(x)$ must be limited between 0 and 1 :

$$
\begin{aligned}
& 0 \leq x \leq 1 \\
& 0 \leq f(x) \leq 1 \\
& 0 \leq g(x) \leq 1
\end{aligned}
$$

Sections of PWL function can be seen in Figure 1. Where J(n)'s are slopes of the independent linear functions, $x n$ 's are x-axis points that divide the function $f(x)$ to functions $g(x)$. Function $f(x)$ and parameter $x$ varies from 0 to 1 .

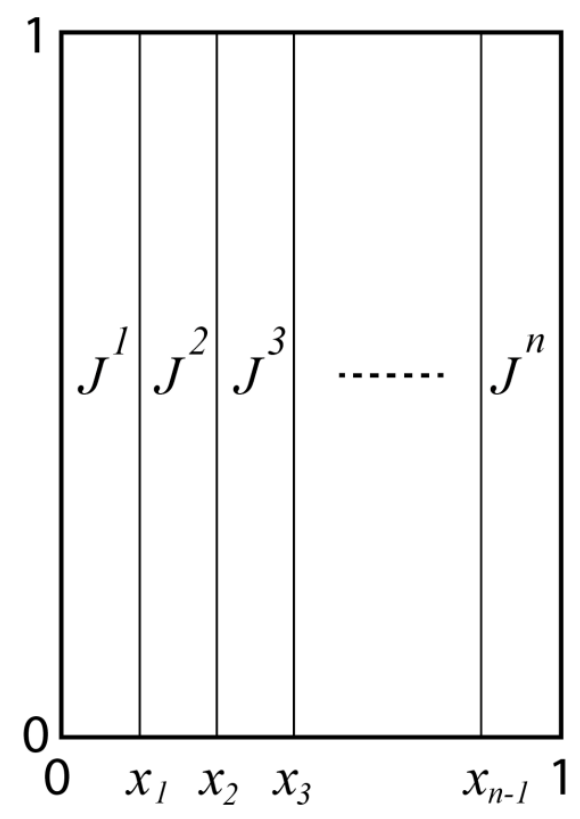

Figure 1. The sections of the memristive element whose length is normalized to 1 using $x=w / D$

The PWL function approximation using functions $g n(x)$ to the function $f(x)$ can be seen in Figure 2 . Functions $g n(x)$ are separated linear functions. If number of functions $g n(x)$ is high enough, function approaches to $f(x)$.

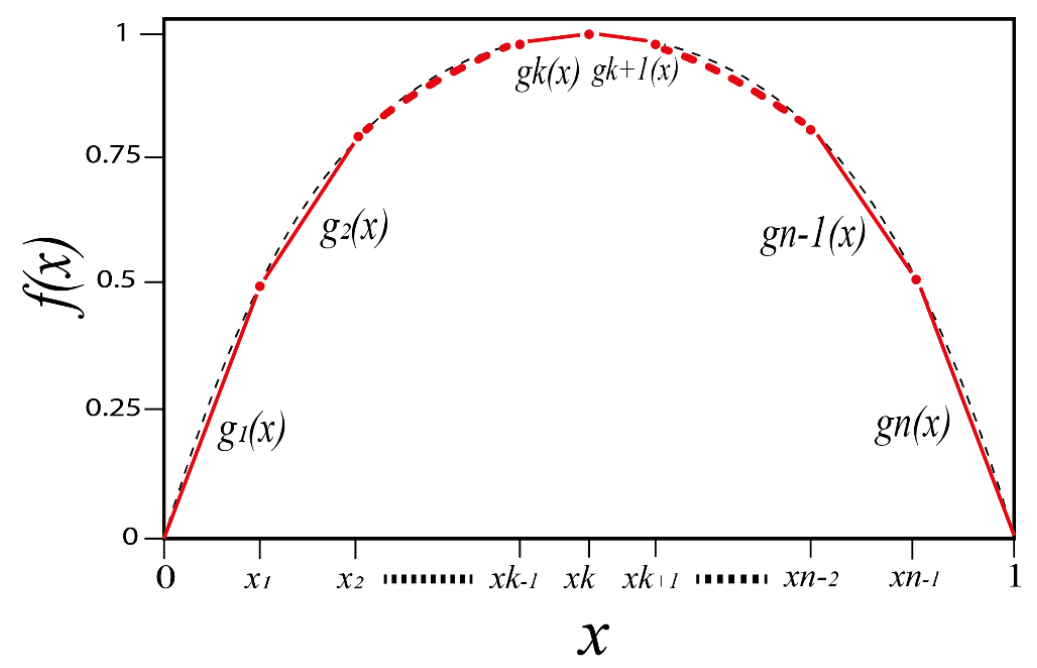

Figure 2. The PWL function approximation $g(x)$ to the function $f(x)$ 
If we know slope and coordinate of one point of the line function, the formula of the function can be written as the following. The PWL function $g(x)$ for the kth segment is given as,

$$
g(x)=\left\{m_{k}\left(x-x_{k}\right)+f\left(x_{k}\right), \quad x_{k} \leq x \leq x_{k+1} .\right.
$$

The tangent (slope) of the PWL function for the kth segment is,

$$
m_{k}=\frac{f\left(x_{k+1}\right)-f\left(x_{k}\right)}{x_{k+1}-x_{k}} \text {. }
$$

If the number of intervals is chosen high enough, it can be assumed that,

$$
f(x) \cong g(x) \text {. }
$$

Some memristor current-voltage hysteresis curve are asymmetric. This requires current dependent window functions. A different window function can be chosen for each current direction. Various number of segmented functions can be used for the PWL window functions. Four segmented PWL functions $f a(x)$ and $f b(x)$ are chosen for positive and negative polarity respectively and shown in Figure 3. They are chosen as symmetric functions with respect to the axis of $x=0.5$ that it is used to show the current direction dependency of the memristor.

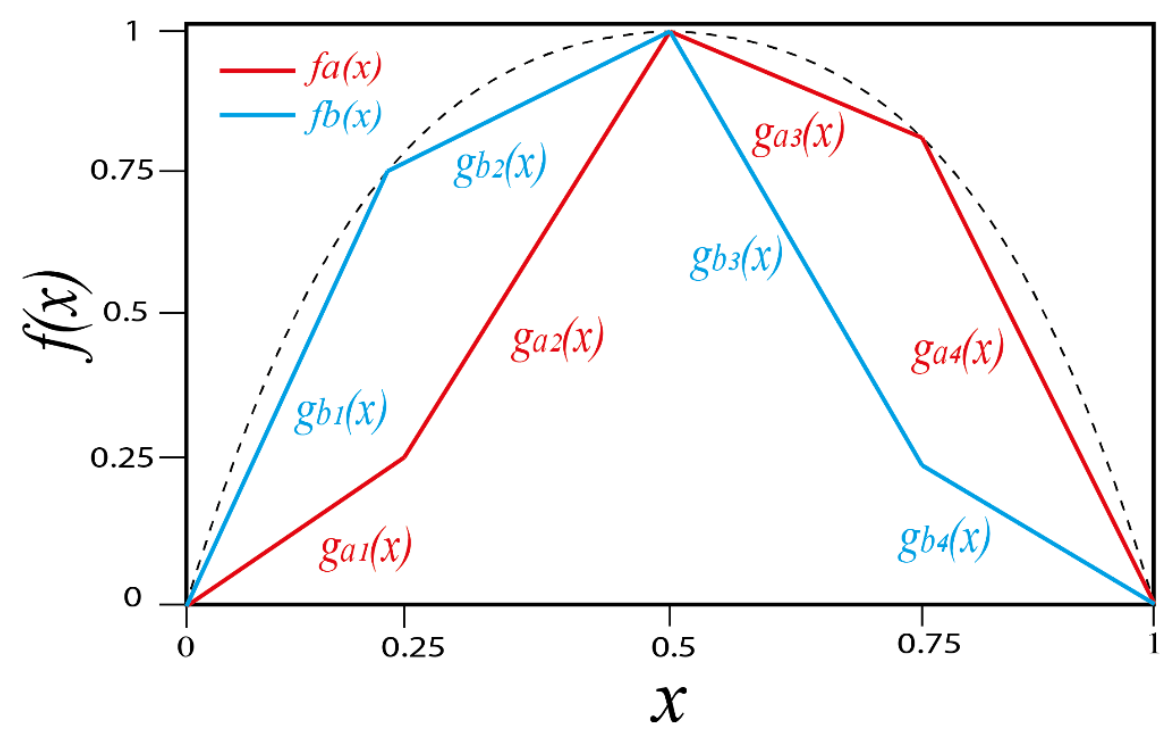

Figure 3. Window functions $f_{a}(x)$ and $f_{b}(x)$

Segments of the PWL functions can be expressed as,

$f(x)= \begin{cases}g_{1}(x), & 0 \leq x<0.25 \\ g_{2}(x), & 0.25 \leq x<0.5 \\ g_{3}(x), & 0.5 \leq x<0.75 \\ g_{4}(x), & 0.75 \leq x \leq 1\end{cases}$

where $f(x)$ is window function, $g_{n}(x)$ are linear functions. The function $\mathrm{A}, f_{a}(x)$, in figure 3 can be given as,

$f_{a}(x)=\left\{\begin{array}{cc}x & 0 \leq x<0.25 \\ 3 x-0.5 & , \quad 0.25 \leq x<0.5 \\ -x+1.5, & 0.5 \leq x<0.75 \\ -3 x+3 & , \quad 0.75 \leq x \leq 1\end{array}\right.$ 
Window function $\mathrm{B}, f_{b}(x)$, is determined as symmetric of function $\mathrm{A}, f_{a}(x)$, with respect to axis $\mathrm{x}=0,5$. Function B as segmented function can be seen,

$f_{b}(x)=\left\{\begin{array}{cc}3 x, & 0 \leq x<0.25 \\ x+0.5, & 0.25 \leq x<0.5 \\ -3 x+2.5, & 0.5 \leq x<0.75 \\ -x+1, & 0.75 \leq x \leq 1\end{array}\right.$.

Window function A taken from [11] can be written using Chua's PWL function as,

$f_{a}(x)=1.5-x+|x-0.25|-2|x-0.5|-|x-0.75|$.

Window function B can be written using Chua's PWL function as,

$f_{b}(x)=0.5+x-|x-0.25|-2|x-0.5|+|x-0.75|$.

Biolek's window function has two variables which are state variable $\mathrm{x}$ and current $i(t)$ of the memristor [13]. In Biolek's model, current direction of the memristor used to define boundary behavior of the window function or to solve boundary tackling issues. When the current direction of memristive device reversed at any moment, ions starts moving the opposite direction with a different velocity regardless of its past. This is how Biolek's window function resolve boundary tackling issues. By taking inspiration from Biolek's window function, the current-dependent window function can be written as,

$f(x, i)=\operatorname{stp}(i) f_{a}(x)+(1-\operatorname{stp}(i)) f_{b}(x)$

where $\operatorname{stp}()$ is the unit step function, which is described as,

$\operatorname{stp}(i)= \begin{cases}1, & i \geq 0 \\ 0 & , \quad i<0\end{cases}$

As seen in eq. (21), the new window function has two independent PWL window functions selected depending on the current direction of the memristive element. For $i \geq 0, f a(x)$ and $i<0, f b(x)$ are used respectively as the window functions. Providing that $f a(0)=0$ for $i(t)>0$ and $f b(1)=0$ for $i(t)<0$, the boundary tackling issues for memristors are solved. In other words: If $g 1(0), g 1(1)$ and $g 2(0), g 2(1)$ are different than zero, boundary tackling issue are solved.

\section{MEMRISTOR SPICE MODEL WITH THE PWL WINDOW FUNCTIONS}

Models have been composed using LTSpice simulator environment because it is free of charge and most of the memristor models have been composed using it. LTSpice code of the model is given in the Table 2 . Spice codes are written for both formats of PWL functions. Memristor block scheme is shown in Figure 4. A current source is used to represent memristor and another current source and a capacitor used to calculate state variable. This memristor model has 3 pins. Pin S is used to plot the state variable of the memristor.

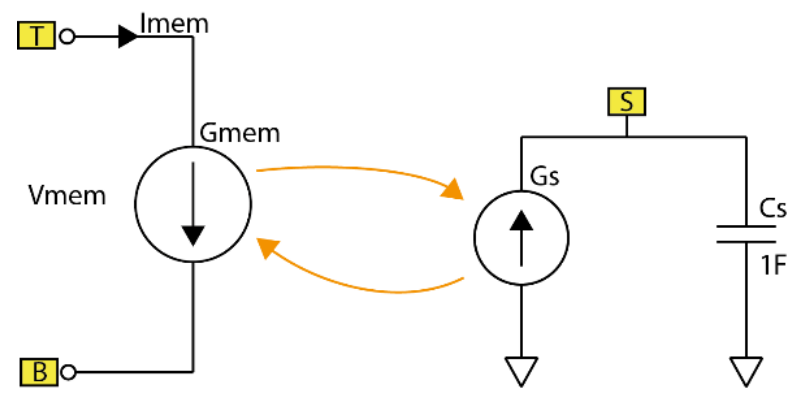

Figure 4. Block Scheme of Memristor Model 
Table 2. The Spice code of the current-dependent PWL memristor model

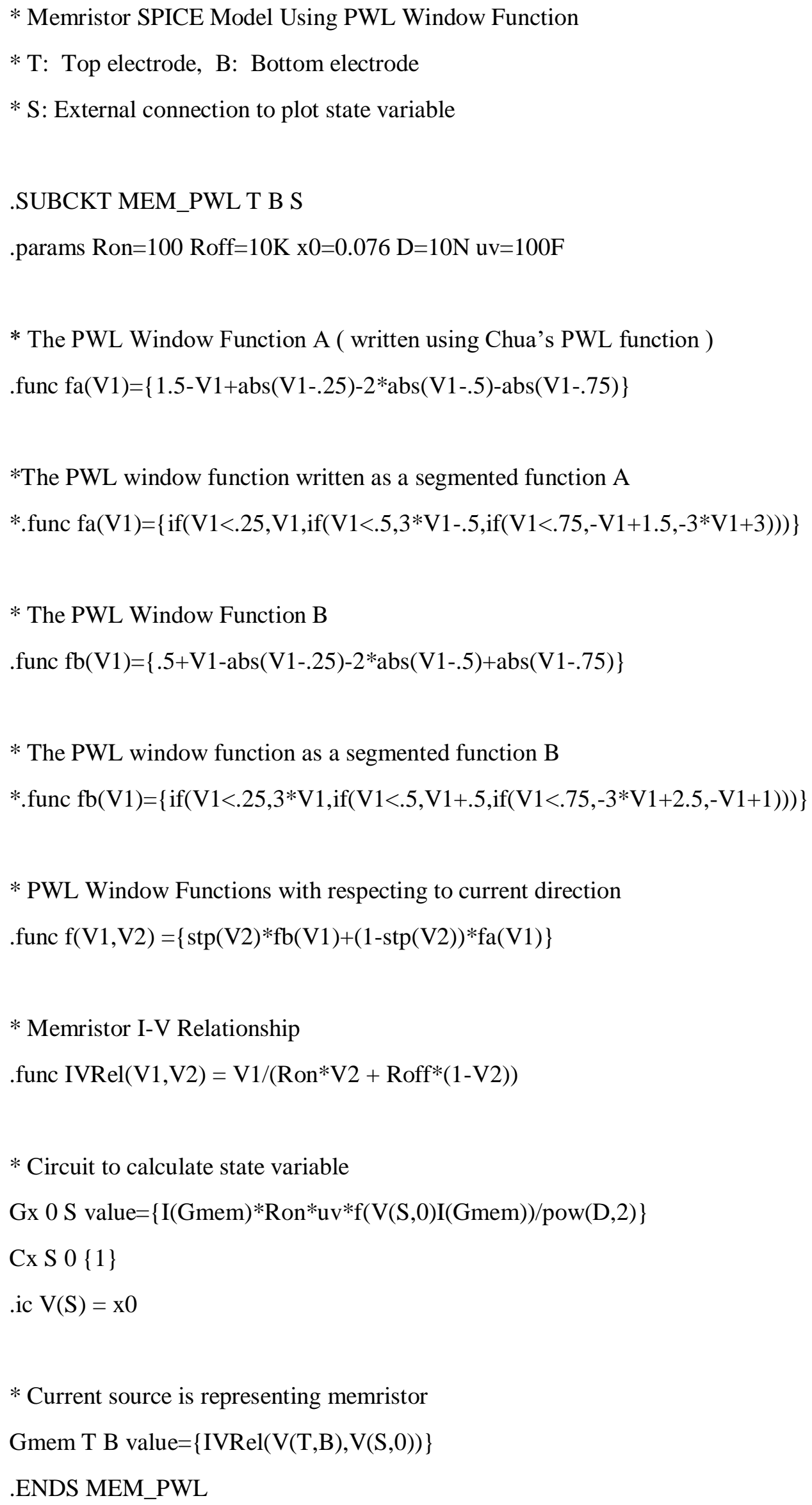




\section{SIMULATION RESULTS OF SPICE MODEL}

In this section, the simulation results for the PWL memristor model given in [21] and the current-polarity dependent memristor model are simulated, results are given. Then, the PWL window functions named A and B are used to make current polarity-dependent window function of the suggested memristor model. LTSpice model codes are written for both PWL function formats as shown in Table 2. Each memristor model has been simulated using with sinusoidal signals of three different frequencies $(10 \mathrm{~Hz}, 12 \mathrm{~Hz}$ and $20 \mathrm{~Hz}$ ) in LTSpice. Voltage-current curves of the models and voltages, currents of memristances, and state variables of the PWL memristor models are also given. Simulation parameters of memristor model can be seen in LTSpice code of the memristor model.

Voltage-current curves of PWL memristor model given in [21] with window function A (without currentpolarity dependency) can be seen in Figure 5. Window function A has been taken from the research of Hernández-Mejía et al. [21]. The model has been simulated for three different frequencies: $10 \mathrm{~Hz}, 12 \mathrm{~Hz}$ and $20 \mathrm{~Hz}$. Frequency dependence of the model is shown by the simulation results given in Figure 5. Voltage, current, memristance and state variable curves of PWL memristor model suggested in [21] with window function A have been shown in Figure 6. Over the $20 \mathrm{~Hz}$, the model starts behaving as if time-invariant resistor.

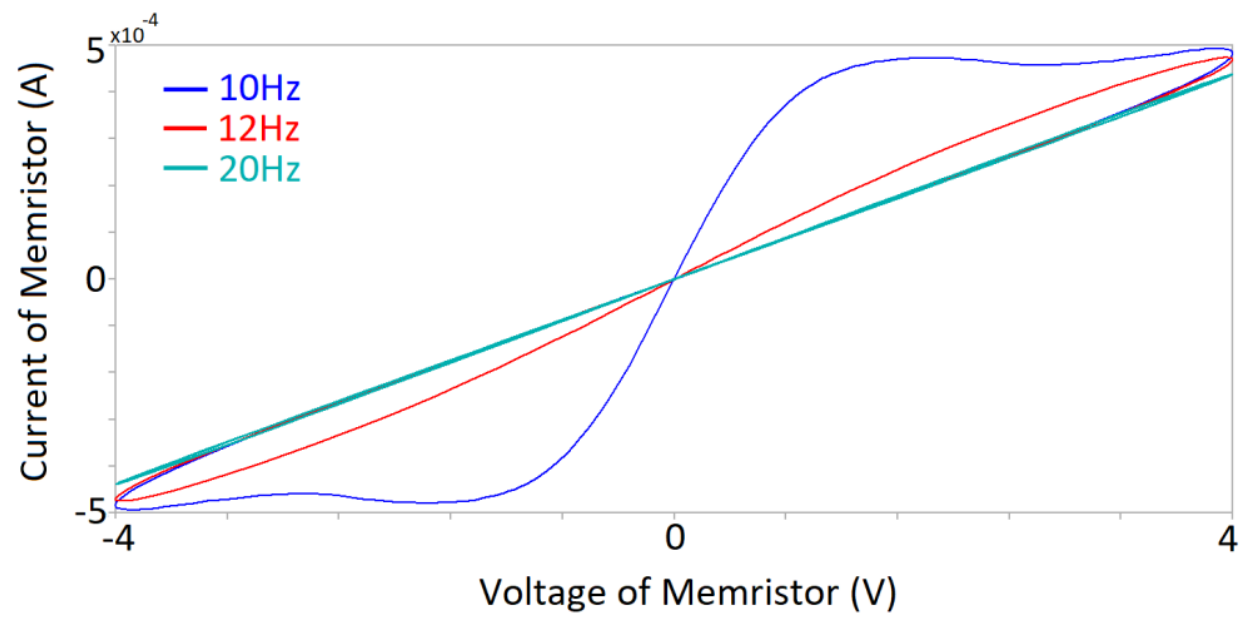

Figure 5. Voltage-current curves of PWL memristor model (without current-polarity dependency) with the window function $A$

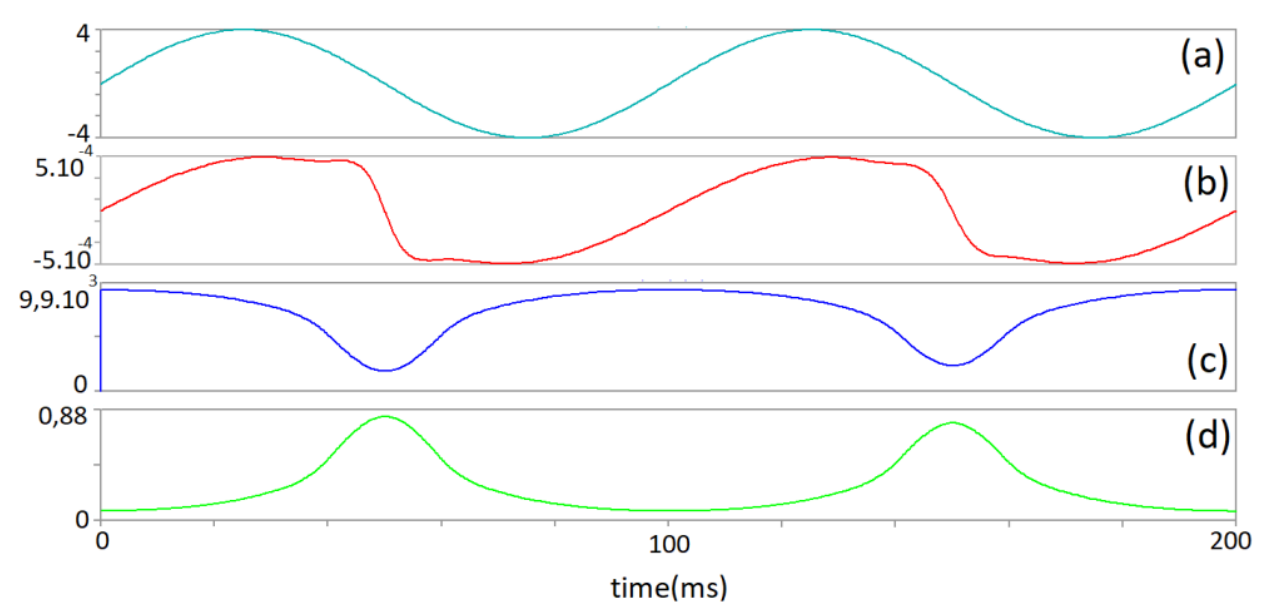

Figure 6. Voltage, Current, Memristance and State variable curves of PWL memristor model (without current-polarity dependency) with the window function A. a) Voltage of memristor, b) Current of memristor, c) Memristance of memristor, d) State variable of memristor 
Voltage-current curves of PWL memristor model with window function B (without current-polarity dependency) can be seen in Figure 7.

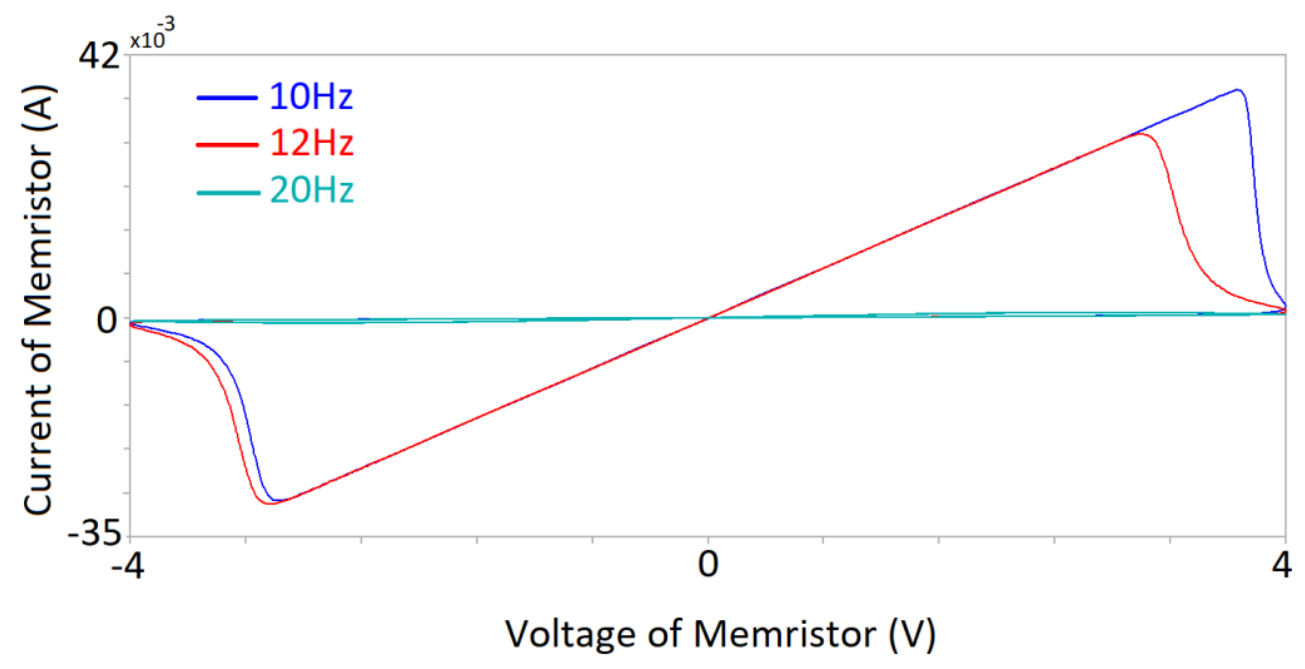

Figure 7. Voltage-current curves of PWL memristor model (without current-polarity dependency) with window function $B$

Voltage, current, memristance and state variable curves of PWL memristor model with window function B (without current-polarity dependency) are shown in Figure 8.

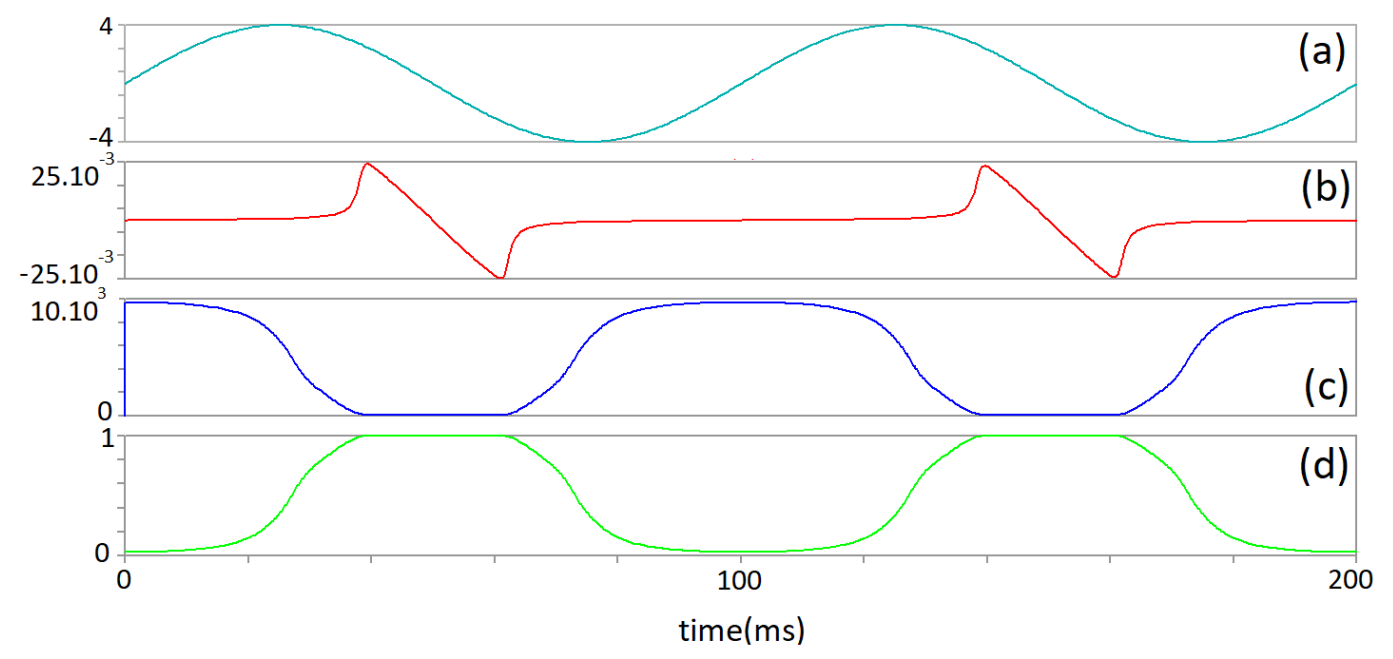

Figure 8. Voltage, Current, Memristance and State variable curves of PWL memristor model (without current-polarity dependency) with window function B. a) Voltage of memristor, b) Current of memristor, c) Memristance of memristor, d) State variable of memristor

Voltage-current curves of the current polarity-dependent PWL memristor model suggested in this paper is shown in Figure 9. The current dependency of the model can easily be seen in Figure 9. Voltage, current, memristance and the state variable curves of the current polarity-dependent PWL memristor model are shown in Figure 10. It should be paid attention to state variable of the memristor model, it behaves differently while approaching to and going away from the boundaries of the memristive element. The simulated figures show that using different PWL memristor models can be used to obtain different memristor behaviors easily and it has a potential to model not only unipolar but also bipolar memristive systems. 


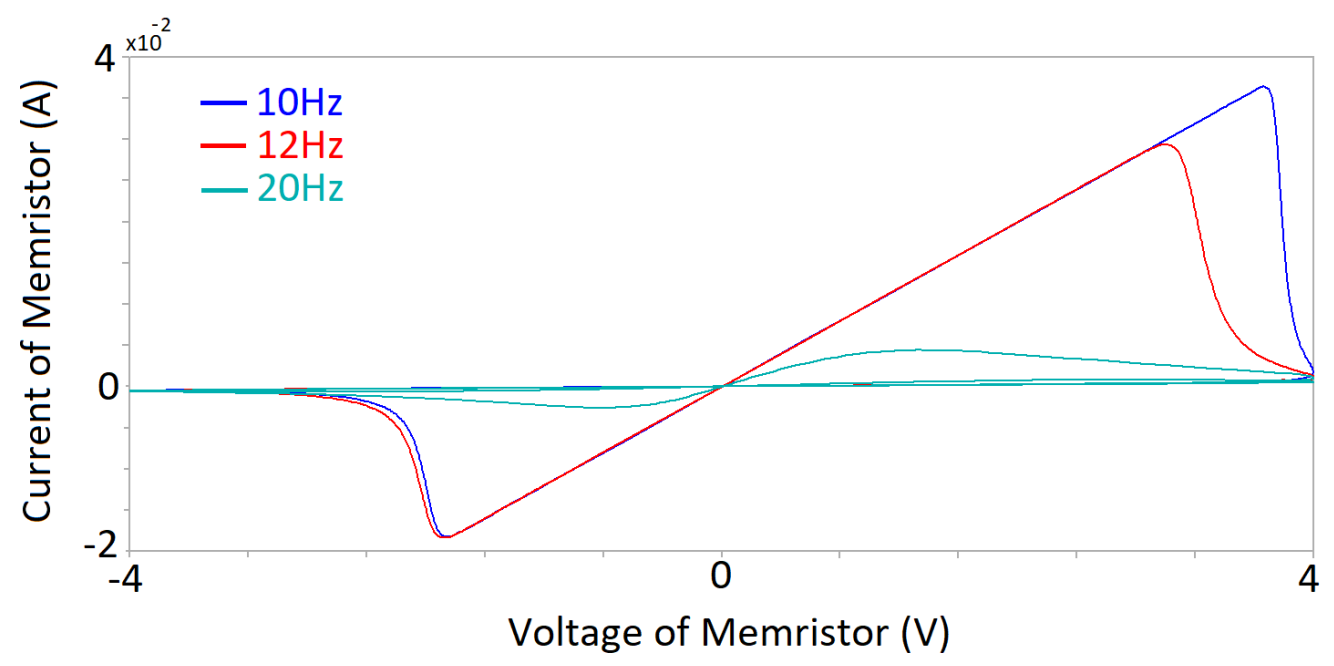

Figure 9. Voltage-current curves of the Current polarity-dependent PWL memristor model

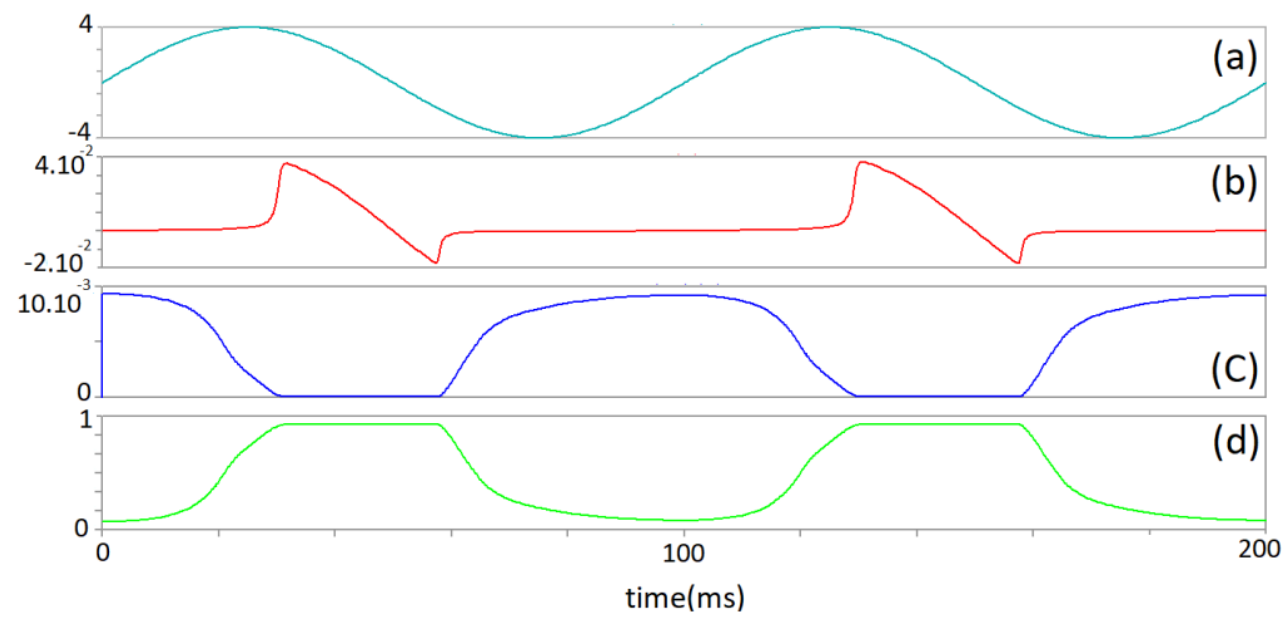

Figure 10. Voltage, Current, Memristance and State variable curves of the Current polarity-dependent $P W L$ memristor model. a) Voltage of memristor, b) Current of memristor, c) Memristance of memristor, d) State variable of memristor

\section{CONCLUSIONS}

In this paper, a current polarity dependent memristor model is proposed. The model makes use of a different PWL window function for each current direction. Another PWL function format which uses line equation for each segment instead of using absolute value like Chua's PWL function does is also given here. It is shown how to resolve the boundary tackling issues by using proper PWL functions for each direction. The required PWL functions whose formulas are also calculated and given. Their Spice model has also been established for both Chua's and the new PWL function's formats. Memristor models with novel window functions are simulated. Simulations are performed for two independent four segmented PWL functions. Voltage, current, memristance and state variable of memristor models simulated are given to examine the model behavior. Using simulations, it is shown that the use of current-polarity dependent PWL functions can model memristor systems more accurately using sufficient number of segments and it is also able model asymmetric hysteresis loops. 


\section{REFERENCES}

[1] Chua, L. O., "Memristor-The missing circuit element", IEEE Trans. Circuit Theory, 18: 507-519, (1971).

[2] Chua, L. O., Kang, S. M., "Memristive devices and systems", Proc. IEEE, 64: 209-223, (1976).

[3] Strukov, D. B., Snider, G. S., Stewart, D. R., Williams, R. S., "The missing memristor found”, Nature (London), 453: 80-83, (2008).

[4] Prodromakis, T., Toumazou, C., "A review on memristive devices and applications", 17th IEEE International Conference on Electronics, Circuits and Systems, 934-937, (2010).

[5] Pershin, Y.V., Martinez-Rincon, J., Di Ventra, M., "Memory circuit elements: from systems to applications", Journal of Computational and Theoretical Nanoscience, 8(3): 441-448, (2011).

[6] Pershin, Y. V., Di Ventra, M., "Memory effects in complex materials and nanoscale systems", Adv. Phys., 60: 145-227, (2011).

[7] Chua, L., "Resistance switching memories are memristors", Appl. Phys. A, 102: 765-783, (2011).

[8] Marani, R., Gennaro, G., Perri, A. G., "A Review on Memristor Applications", International Journal of Advances in Engineering \& Technology, 8(3): 294, (2015).

[9] Sangho, S., Kim, K., Kang, S-M., "Memristor applications for programmable analog ICs.", IEEE Transactions on Nanotechnology, 10(2): 266-274, (2011).

[10] Joglekar, Y. N., Wolf, S. J., "The elusive memrisor: properties of basic electrical circuits", European Journal of Physics, 30(4): 661675, (2009).

[11] Prodromakis, T., Peh, B. P., Papavassiliou, C., Toumazou, C., “A Versatile Memristor Model With Nonlinear Dopant Kinetics”, IEEE Transactions on Electron Devices, 58(9): 3099-3105, (2011).

[12] Oğuz, Y., Gül, F., Eroğlu, H., “A New Window Function for Memristor Modeling”, 8th International Advanced Technologies Symposium, (2017).

[13] Biolek, Z., Biolek, D., Biolkova, V., "SPICE model of memristor with nonlinear dopant drift", Radio Engineering, 18(2): 210-214, (2009).

[14] Zha, J., Huang, T., Liu, Y., "A Novel Window Function for Memristor With Application in Programming Analog Circuits”, IEEE TCAS-II, 63(5): 423-427, (2016).

[15] Itoh, M., Chua, L. O., "Memristor Oscillators", Int. J. Bifurcation and Chaos, 18: 3183-3206, (2008).

[16] Wang, D., Hu, Z., Yu, X., and Yu, J., "A PWL model of memristor and its application example", 2009 International Conference on Communications, Circuits and Systems, 932-934, (2009).

[17] Yener, S. C., Mutlu, R., Kuntman, H. H., "Analysis of filter characteristics based on PWL Memristor", Istanbul University-Journal of Electrical \& Electronics Engineering, 14(1): 1709-1719, (2014).

[18] Güler, H., Kaya, T., "Parça Parça Lineer Memristor Tabanlı Chua Osilatorunun LabVIEW'de Gerçekleştirilmesi”, Frrat Üniversitesi Mühendislik Bilimleri Dergisi, 28(2): 29-33, (2016). 
[19] Solak, A., Herdem, S., “A Piece Wise Linear Memristor Model with Switches,” International Journal of Modeling and Optimization, 6(2): 124, (2016).

[20] Solak, A., Herdem, S., "Simulink Model for Piece Wise Linear Approximation of Memristor", International Journal of Applied Mathematics, Electronics and Computers, 4(1): 386-390, (2016).

[21] Hernández-Mejía, C., Delia T.-M. "PWL Window Function for Nonlinear Memristive Systems." 2019 International Conference on Electronics, Communications and Computers (CONIELECOMP). IEEE, 2019.

[22] Chua, L.O., Kang, S. M., "Section-wise piecewise-linear functions: Canonical representation, properties, and applications", Proceedings of the IEEE, 65: 915-929, (1977). 